\title{
The clinical manifestations at the onset of antisynthetase syndrome: A chameleon with multiple faces
}

\author{
A.C.C.D. Baccaro, G.L. Behrens Pinto, R.C.S. Carboni, S.K. Shinjo \\ Division of Rheumatology, Faculdade de Medicina FMUSP, Universidade de Sao Paulo, SP, Brazil
}

\begin{abstract}
SUMMARY
The antisynthetase syndrome (ASS) is clinically characterized by fever, myositis, interstitial lung disease, joint involvement, mechanic's hands, or Raynaud's phenomenon, and the presence of antisynthetase autoantibodies. These clinical manifestations may not occur simultaneously. Therefore, the aim of this study was to analyze the sequence in which these clinical manifestations can develop at the onset of ASS.

This retrospective, single-center cohort study enrolled 55 ASS patients. Their mean age at the onset of ASS symptoms was $42.3 \pm 11.8$ years. There was a predominance of female patients $(75.9 \%)$ and white patients (72.7\%). At initial presentation, $41.8 \%$ of the patients had fever, $43.6 \%$ had joint symptoms, $38.2 \%$ had myositis, $36.4 \%$ had interstitial lung disease, $18.2 \%$ had Raynaud's phenomenon, and $16.4 \%$ had mechanic's hands. Subsequent clinical symptoms emerged at varying time points. In two out of 55 cases, joint, muscle, and lung manifestations developed simultaneously. The median time between the onset of symptoms and the complete ASS clinical manifestation was 19.9 (4.0-60.2) months; whereas, the timeframe between the onset of symptoms and the ASS diagnosis was 29.0 (11.0-63.0) months. The confounding misdiagnoses interfering with the initial diagnosis were polymyositis (52.7\%), dermatomyositis (29.1\%), nonspecific interstitial pneumopathy (23.6\%), rheumatoid arthritis (18.2\%), and others (10.9\%).

Clinical features at the onset of ASS are highly variable. Consequently, confounding factors can lead to significant delays for the final and definitive diagnosis of ASS. Therefore, ASS should be considered a differential diagnosis in patients with initial symptoms of joint, lung, and/or muscle involvements, as well as fever, mechanic's hands, and/or Raynaud's phenomenon manifestations.
\end{abstract}

Key words: Antisynthetase syndrome; clinical manifestations; myositis; systemic autoimmune myopathies.

Reumatismo, 2020; 72 (2): 86-92

\section{INTRODUCTION}

The antisynthetase syndrome (ASS) is a rare systemic autoimmune myopathy characterized by the presence of an autoantibody against specific aminoacyl-transfer RNA synthetases, plus one or more of the following conditions: myositis, interstitial lung disease, arthritis, unexplained and persistent fever, Raynaud's phenomenon, or mechanic's hands $(1,2)$.

Clinical manifestations of ASS may not necessarily all occur simultaneously. However, so far, few studies have analyzed the clinical course of ASS to confirm this information (3-12). Most of these studies analyzed only ASS patients with anti-Jo-1 autoantibody (3, 6-8). Moreover, in their unicentric cohort study, Pinal-Fernandez et al. (13) suggest that different aminoacyltransfer RNA synthetase autoantibodies (ARS) are associated with different clinical patterns, with the anti-PL-7 and anti-PL-12 autoantibodies related to higher prevalence and severity of interstitial lung disease than anti-Jo-1. However, this topic is still a subject for debate, since Cavagna et al. (5) found a similar presentation and evolution, regardless of the specific autoantibody found in a large cohort of ASS patients.

In particular, a multicenter Spanish study that included 148 patients with anti-Jo-1 ASS showed that most patients (77.2\%) have isolated lung, muscle, or joint involvement at the onset of the disease (3). Likewise, an international multicenter 
study by Cavagna et al. (7) showed that most anti-Jo-1 ASS patients have the incomplete clinical form, with arthritis, myositis or lung involvement, at the onset of disease. This study also revealed that these patients evolve to the complete clinical form with all the manifestations of the classical triad in a few months or years (7).

These heterogeneous and incomplete clinical presentations of ASS have become a challenge in clinical practice. This challenge can lead physicians to a wrong diagnosis and, therefore, delay the correct ASS diagnosis and treatment.

Therefore, the aim of the study was to describe the clinical manifestations at the onset of the disease in our ASS Brazilian cohort and then analyze chronologically and longitudinally the development of other symptoms to identify a development pattern of clinical manifestations that could help rheumatologists diagnose early and correctly the disease. In addition, we have described the differential diagnoses made before the definitive ASS diagnosis.

\section{PATIENTS AND METHODS}

This retrospective, unicenter cohort study evaluated 55 consecutive patients with ASS, who were aged more than 18 years, from our tertiary outpatient clinic from 2000 to 2019.

This study was approved by the local Ethics Committee (HCFMUSP, CAAE 68523717.1. 0000.0068) and all participants signed an informed consent form.

The patients included in this study fulfilled the modified classification criteria proposed by Connors et al. (1) and by Cavagna et al. (5). For laboratory criteria, patients should have a serum positive test against one of the following aminoacyl-transfer RNA synthetases: anti-Jo-1, -OJ, -EJ, -PL7, or -PL-12. Clinically, the patients should have all of the following conditions: unexplained and persistent fever; Raynaud's phenomenon; mechanic's hands; and muscle, lung, and joint involvements. Then, we retrospectively reviewed the medical records of these patients for information about patterns of clinical manifestations at the onset of the disease and their evolution over the years of follow-up.

Muscle involvement was defined as the presence of proximal, progressive muscle weakness and increased muscle enzymes (for instance, creatine phosphokinase, or CPK), in addition to electroneuromyography showing a myopathic pattern with or instead of a muscle biopsy compatible with inflammatory myopathy. According to previous publications in $\operatorname{ASS}(5,6,9)$, joint involvement was defined as arthralgia or arthritis (non-erosive). According to the American Thoracic Society (14), pulmonary involvement was defined as the presence of reported dyspnea and altered computed tomography (incipient pneumopathy, ground-glass opacities with or without bronchiectasis or pulmonary fibrosis and honeycombing areas).

The antisynthetase autoantibodies (antiJo-1, anti-PL-7, anti-PL-12, anti-OJ, antiEJ) and myositis-associated autoantibodies (anti-PM/Scl, anti-Ku, anti-Ro-52) were identified by a commercially available line blot test kit (Myositis Profile Euroline Blot test kit, Euroimmun, Lübeck, Germany). The assessment was performed according to previously established methods (15).

The exclusion criteria included overlapped autoimmune diseases, cancer-associated myositis, and patients with anti-PM/Scl or anti-Ku autoantibodies. We excluded also patients with double positivity to myositisspecific autoantibodies.

Patient records were extensively reviewed for the following data:

a) Demographic data, including age at onset of symptoms, age at ASS diagnosis, gender, and ethnicity;

b) Cumulative clinical manifestations or onset of symptoms, including fever, weight loss, muscle weakness graduated according to the Medical Research Council Scale (16), joint involvement, dyspnea, Raynaud's phenomenon, and mechanic's hands;

c) Laboratory criteria, including initial serum levels of creatine phosphokinase (reference value: 26 - 192 U/L);

d) Autoantibody analyses, including antiJo-1, anti-PL-7, anti-PL-12, anti-OJ, 
anti-EJ, anti-Ro-52, anti-PM/Scl, and anti-Ku; and

e) Initial differential diagnosis.

The patients' initial symptoms were classified into first, second, and third manifestations, according to the manifestations of the symptoms over time.

\section{Data}

The Kolmogorov-Smirnov test was used to evaluate the distribution of each parameter. The demographic and clinical features were expressed as mean \pm standard devia-

Table I - General features of the 55 patients with antisynthetase syndrome.

\begin{tabular}{|l|c|}
\hline Age at & \\
Symptoms' onset (years) & $42.3 \pm 11.8$ \\
ASS diagnosis (years) & $46.0 \pm 13.1$ \\
Female gender (\%) & $42(75.9)$ \\
White ethnicity (\%) & $40(72.7)$ \\
\hline Initial cumulative manifestations & \\
Weight loss (\%) & $49(89.1)$ \\
Fever (\%) & $55(100.0)$ \\
Muscle involvement (\%) & $55(100.0)$ \\
Upper limb strength & $1(1.8)$ \\
Degree I & $1(1.8)$ \\
Degree II & $15(27.3)$ \\
Degree III & $31(56.8)$ \\
Degree IV & $7(12.7)$ \\
Degree V & \\
Lower limb strength & 0 \\
Degree I & $1(1.8)$ \\
Degree II & $16(29.1)$ \\
Degree III & $37(67.3)$ \\
Degree IV & $1(1.8)$ \\
Degree V & $55(100.0)$ \\
Joint involvement (\%) & $55(100.0)$ \\
Arthralgia (\%) & $38(69.1)$ \\
Arthritis (\%) & $55(100.0)$ \\
Lung involvement (\%) & $55(100.0)$ \\
Dyspnea (\%) & $55(100.0)$ \\
Raynaud's phenomenon & $55(100.0)$ \\
"Mechanic's hands" & $46(83.6)$ \\
\hline Maximum serum level of creatine phosphokinase (U/L) & $4838(1298-9057)$ \\
\hline Lung involvement (\%) & $35(100.0)$ \\
Computer tomography & $3(5.5)$ \\
Incipient pneumopathy & $5(100.0)$ \\
Ground-glass opacities & $32(58.2)$ \\
Pulmonary fibrosis & $11(20.0)$ \\
\hline Autoantibodies & \\
Anti-Jo-1 (\%) & \\
Anti-PL-7 (\%) & \\
Anti-PL-12 (\%) & \\
Anti-EJ (\%) & \\
Anti-OJ (\%) & \\
Anti-Ro-52 (\%) & \\
\hline
\end{tabular}

tions for the continuous variables or as frequencies $(\%)$ for the categorical variables. The median $\left(25^{\text {th }}-75^{\text {th }}\right.$ interquartile range $)$ was calculated for continuous variables that were non-normally distributed. All analyses were performed using the SPSS 15.0 statistics software (Chicago, IL, USA).

\section{RESULTS}

Fifty-five ASS patients met the inclusion criteria and were evaluated. The means of the patients' ages at the onset of symptoms and at the ASS diagnosis were $42.3 \pm 11.8$ and $46.0 \pm 13.1$ years, respectively. There was a predominance of female $(75.9 \%)$ and white patients $(72.7 \%)$. Table I shows the initial and general cumulative features of the ASS patients.

At presentation, $41.8 \%$ of the cases had fever, $43.6 \%$ had joint symptoms, $38.2 \%$ had myositis, $36.4 \%$ had interstitial lung disease, $18.2 \%$ had Raynaud's phenomenon, and $16.4 \%$ had mechanic's hands. Subsequent clinical symptoms emerged at different timepoints (Figure 1).

The second symptoms were muscle involvement in $38.2 \%$ of the cases, Raynaud's phenomenon in $31.7 \%$, lung involvement in $32.7 \%$, joint involvement in $27.3 \%$, fever in $25.5 \%$, and mechanic's hands in $25.5 \%$.

The third symptoms were mechanic's hands in $36.4 \%$ of the patients, Raynaud's phenomenon in $30.9 \%$, lung involvement in $21.8 \%$, joint involvement in $20.0 \%$, fever in $18.2 \%$, and muscle involvement in $16.4 \%$. The joint, muscle, and lung symptoms appeared simultaneously in only two $(2.6 \%)$ of the 55 cases.

Subsequent clinical symptoms emerged at different timepoints, leading to variety of diagnoses and therapeutic schemes. The median time between the onset of symptoms and the complete ASS clinical manifestation was 19.9 (4.0-60.2) months; whereas, the mean diagnostic delay (time between ASS diagnosis and the onset of symptoms) was 29.0 (11.0-63.0) months. During the period between the ASS diagnosis and the onset of symptoms, $51(92.7 \%)$ 


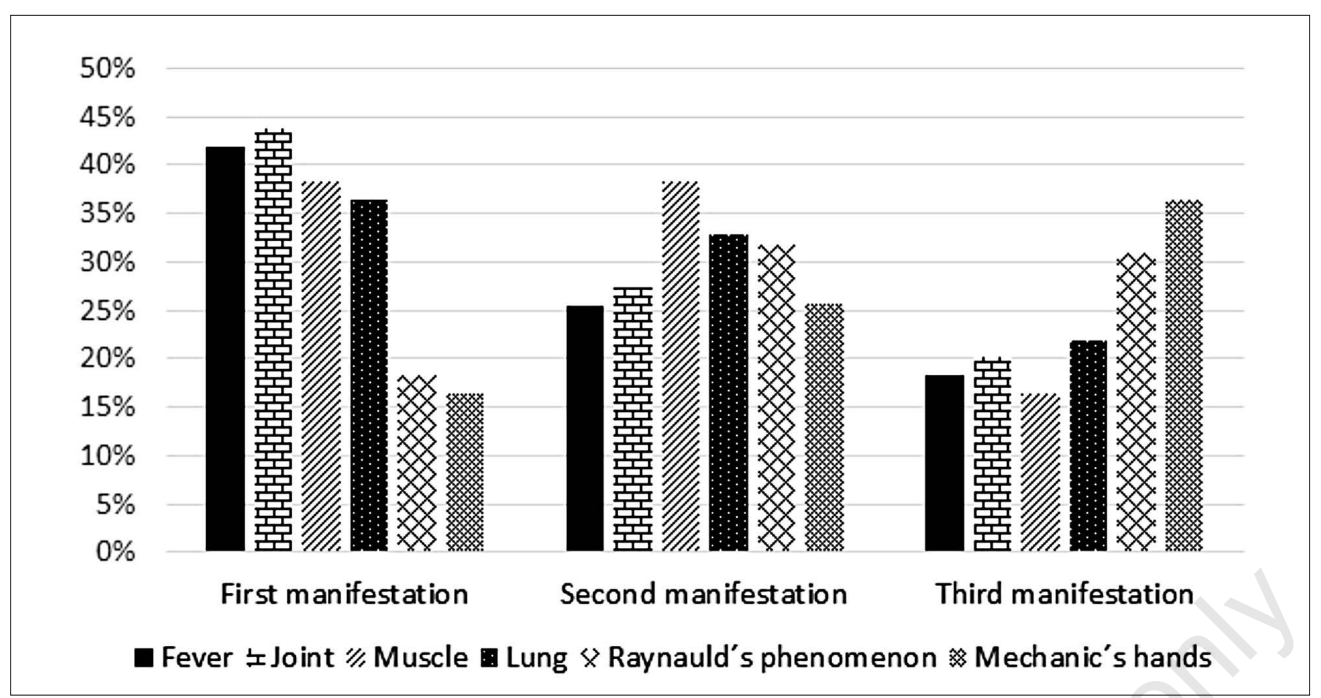

Figure 1 - Subsequent clinical symptoms in the 55 patients with antisynthetase syndrome.

of the 55 patients received glucocorticoids and/or immunosuppressive drugs.

Due to incomplete and varied clinical manifestations, the confounding diseases in the initial diagnosis were polymyositis $(52.7 \%)$, dermatomyositis $(29.1 \%)$, rheumatoid arthritis $(18.2 \%)$, non-specific interstitial pneumopathy (23.6\%), or idiopathic pulmonary interstitial fibrosis $(9.1 \%)$. In 21 (38.2\%) of the 55 cases, concomitant diagnostic hypotheses (as mentioned above) were performed (Table II).

\section{DISCUSSION AND CONCLUSIONS}

We described the clinical manifestations of ASS at the beginning of the disease and then chronologically and longitudinally analyzed the onset of new symptoms through the years of follow-up. Fever, joint, and muscular symptoms predominated as the initial symptoms; whereas mechanic's hands, Raynaud's phenomenon, and pulmonary manifestations appeared afterwards. Moreover, due to the temporal variability of the manifestation of symptoms and the incomplete manifestation of symptoms, other diagnoses were erroneously made, delaying the ASS diagnosis.

In this study, we included patients according to the modified classification criteria
Table II - Initial diagnosis of the 55 patients with antisynthetase syndrome.

\begin{tabular}{|l|c|}
\hline Polymyositis (\%) & $29(52.7)$ \\
Dermatomyositis (\%) & $16(29.1)$ \\
Non-specific interstitial pneumopathy (\%) & $13(23.6)$ \\
Rheumatoid arthritis (\%) & $10(18.2)$ \\
Idiopathic pulmonary interstitial fibrosis (\%) & $5(9.1)$ \\
Others (\%) & $6(10.9)$ \\
Systemic lupus erythematosus (\%) & $2(3.6)$ \\
Bronchopneumonia (\%) & $2(3.6)$ \\
Sjögren's syndrome (\%) & $1(1.8)$ \\
Systemic sclerosis (\%) & $1(1.8)$ \\
\hline
\end{tabular}

In 21 (38.2\%) of the 55 cases, concomitant diagnostic hypotheses (as mentioned above) were performed.

proposed by Connors et al. (1) and Cavagna et al. (5) to increase specificity and avoid possible selection biases. Despite this rigorous selection criterion, we included a large sample of patients with ASS, which is considered a rare systemic autoimmune disease and is scarcely described in the literature.

The patients' ages at the onset of symptoms and the ASS diagnosis were 42.3 and 46.0 years, respectively. There was a predominance of female and white patients. Therefore, our data are consistent with the analysis of 813 ASS patients conducted by the American and European Network of Antisynthetase Syndrome (AENEAS) study (5) that showed a prevalence of female patients $(74 \%)$ and a higher mean age at the onset of disease symptoms (51 years). 
Cavagna et al. (7) noted in their multicenter retrospective study of 255 patients with ASS that most patients did not initially present with the classic triad of disease manifestations (arthritis, myositis, and interstitial lung disease), but with only one of these symptoms. They also showed that these patients often developed the complete form of the disease during the followup period and that the development of different manifestations can take from months to years. In another retrospective multicenter study, Cavagna et al. (17) concluded that the classic triad is not commonly observed, and fever, mechanic's hands, or Raynaud's phenomenon may be predictors for the onset of at least one of the classic triad symptoms in the anti-Jo-1 positive ASS patients. In our study, however, fever was a frequent first manifestation, along with joint and muscular symptoms, whereas pulmonary involvement, mechanic's hands, and Raynaud's phenomenon usually appeared over the course of the disease. Also in agreement with our study, González-Gay et al. (11) demonstrated that joint involvement usually happens at the beginning of the disease, and to a lesser extent during follow-up.

On the contrary, Trallero-Araguás et al. (3) concluded in their retrospective multicenter study that myositis, arthritis, and interstitial lung disease were the most frequent initial symptoms of ASS. They also demonstrated a variation between the time of onset of symptoms and the diagnosis of ASS, depending on the initial organ involvement manifestations. They concluded that patients with isolated arthritis had the longest diagnostic delay compared to the other groups analyzed (ILD plus myositis, ILD without myositis, isolated myositis). The mean delay in diagnosis in patients with isolated arthritis was 24 months, a much longer period than in the other groups (where it was less than 12 months) (3).

This pattern of onset with isolated symptoms and incomplete forms of the disease as well as the similarity of ASS to other autoimmune diseases can result into possible diagnostic mistakes for patients with ASS. Alves et al. (18) observed that patients with dermatomyositis and polymyositis with the presence of autoantibodies anti-PM/Scl demonstrated a pattern of clinical presentation similar to that of ASS, confusing the diagnosis, which is the reason we also elected to exclude patients with anti-PM/ Scl autoantibodies in this study.

When a patient presents with joint involvement, the pattern is a peripheral symmetric polyarthritis, similar to that of rheumatoid arthritis (19). The patient may also present with rheumatoid factor, anti-cyclic citrullinated peptide (anti-CCP) autoantibodies, and bone erosions that may be correlated with anti-CCP positivity $(19,20)$. When patients have isolated joint symptoms, it is difficult for rheumatologists to think about ASS. Indeed, 10 (18.2\%) of our patients were diagnosed with rheumatoid arthritis, before they received the correct diagnosis of ASS. Cavagna et al. (7) concluded that a test for the anti-Jo-1 autoantibody should be ordered in any patient with arthritis, isolated myositis, or interstitial lung disease, as its positivity could predict clinical occurrences during follow-up and could change the diagnosis. Therefore, it is reasonable to perform periodic screenings for myositis and interstitial lung diseases, even in patients with more likely diagnoses of rheumatoid arthritis (19).

When pulmonary involvement occurs without other clinical manifestations, the diagnosis of non-specific interstitial lung disease or idiopathic pulmonary fibrosis may be considered when ASS is not suspected (4, 6). Giannini et al. (21) suggested in their publication of three case reports that the test for myositis-specific antibodies should be performed in patients with idiopathic interstitial lung disease, even without other extrapulmonary manifestations.

This study had some limitations. It was a retrospective study. In addition, we analyzed patients who were treated only by rheumatologists and were not followed in other specialties, such as, for instance, a patient diagnosed with interstitial lung disease followed only by pulmonologists. Our retrospective design did not it make possible to perform correlations between initial clinical manifestations and the outcomes or 
prognoses. We also do not have information about the medications used between the initial clinical manifestations and the ASS diagnoses in all patients or how long these medications could have interfered with the clinical course of the disease. We did not explore whether different types of aminoacyl-transfer RNA synthetase autoantibodies were associated with different types of onset and disease patterns, because of the small samples in each autoantibody group (except for anti-Jo-1). Finally, we also had problems studying ASS due to the lack of a widely accepted validated classification criteria for the ASS (22).

In conclusion, clinical features at the onset of ASS are highly variable. Our patients presented with fever and muscle and/or joint symptoms as initial indicators; whereas, Raynaud's phenomenon, mechanic's hands, and pulmonary symptoms were late indicators of the disease. Consequently, the confounding factors led to significant delays before reaching the final and definitive diagnosis of ASS.

We believe this analysis will contribute to the early detection and diagnosis of ASS, thus making it possible to offer an appropriate treatment and reduce the morbidity and mortality of the patients.

\section{Funding}

This work was funded by Conselho Nacional de Desenvolvimento Científico e Tecnológico (CNPq) \#303379/2018-9, and Faculdade de Medicina da USP - SP to SKS.

\section{Conflict of interest}

The authors declare no conflict of interest.

\section{REFERENCES}

1. Imbert-Masseau A, Hamidou M, Agard C, Grolleau JY CP. Antisynthetase syndrome. Jt Bone Spine. 2003; 70: 161-8.

2. Connors GR, Christopher-Stine L, Oddis CV, Danoff SK. Interstitial lung disease associated with the idiopathic inflammatory myopathies: What progress has been made in the past 35 years? Chest. 2010; 138: 1464-74.

3. Trallero-araguás E, Grau-junyent JM, Monteagudo-jiménez M, Fraile-rodriguez G. Clini- cal manifestations and long-term outcome of anti-Jo1 antisynthetase patients in a large cohort of Spanish patients Study Group and Autoimmune Diseases Study Group (GEAS ) of the Spanish Society of. Semin Arthritis Rheum. 2016; 46: 225-31.

4. Chatterjee S, Prayson R, Farver C. Antisynthetase syndrome: not just an inflammatory myopathy. Cleve Clin J Med. 2013; 80: 655-66.

5. Cavagna L, Trallero-aragu E, Meloni F, et al. Influence of antisynthetase antibodies specificities on antisynthetase syndrome clinical spectrum time course. J Clin Med. 2019; 8: $1-13$.

6. Bartoloni E, Gonzalez-Gay MA, Scirè C, et al. Clinical follow-up predictors of disease pattern change in anti-Jo1 positive anti-synthetase syndrome: Results from a multicenter, international and retrospective study. Autoimmun Rev. 2017; 16: 253-7.

7. Cavagna L, Nuño L, Scirè CA, et al. Clinical spectrum time course in anti jo-1 positive antisynthetase syndrome: Results from an international retrospective multicenter study. Medicine. 2015; 94: e1144.

8. Shinjo SK, Levy-Neto M. Anti-Jo-1 antisynthetase syndrome. Rev Bras Reumatol. 2010; 50: 496-500.

9. Monti S, Montecucco C, Cavagna L. Clinical spectrum of anti-Jo-1-associated disease. Curr Opin Rheumatol. 2017; 29: 612-7.

10. Sebastiani M, Triantafyllias K, Manfredi A, et al. Nailfold capillaroscopy characteristics of antisynthetase syndrome and possible clinical associations: results of a multicenter international study. J Rheumatol. 2019; 46: 279-84.

11. González-Gay MA, Montecucco C, SelvaO'Callaghan A, et al. Timing of onset affects arthritis presentation pattern in antisyntethase syndrome. Clin Exp Rheumatol. 2018; 36: 44-9.

12. Scirè CA, Gonzalez-Gay MA, SelvaO'Callaghan A, Cavagna L. Clinical spectrum time course of interstitial pneumonia with autoimmune features in patients positive for antisynthetase antibodies. Respir Med. 2017; 132: 265-6.

13. Pinal-Fernandez I, Casal-Dominguez M, Huapaya JA, et al. A longitudinal cohort study of the anti-synthetase syndrome: Increased severity of interstitial lung disease in black patients and patients with anti-PL7 and antiPL12 autoantibodies. Rheumatology (United Kingdom). 2017; 56: 999-1007.

14. Nici L, Donner C, Wouters E, et al. American Thoracic Society/European Respiratory Society statement on pulmonary rehabilitation. Am J Respir Crit Care Med. 2006; 173: 1390-413.

15. Pina Cruellas MG, Trindade Viana V dos S, Levy-Neto M, et al. Myositis-specific and myositis-associated autoantibody profiles and 
their clinical associations in a large series of patients with polymyositis and dermatomyositis. Clinics. 2013; 68: 909-14.

16. Compston A. Aids to the investigation of peripheral nerve injuries. Medical Research Council: Nerve Injuries Research Committee. His Majesty's Stationery Office: 1942; By Michael O'Brien for the Guarantors of Brain. Saunders Elsevier: 2010; pp. [8] 64 and 94 Figures. Brain 2010; 133: 2838-44.

17. Cavagna L, Nuño L, Scirè CA, et al. Serum Jo-1 autoantibody and isolated arthritis in the antisynthetase syndrome: review of the literature and report of the experience of AENEAS Collaborative Group. Clin Rev Allergy Immunol. 2017; 52: 71-80.

18. Alves SP, Silva MG, Borges IBP, Shinjo SK. Patients with pure dermatomyositis/polymyositis and anti-PM/Scl autoantibody resembling anti-synthetase syndrome. Med Express. 2018; 5: 17-9.
19. Ingegnoli F, Galbiati V, Zeni S, et al. Use of antibodies recognizing cyclic citrullinated peptide in the differential diagnosis of joint involvement in systemic sclerosis. Clin Rheumatol. 2007; 26: 510-4.

20. Emad Y, Ragab Y, Abd-Elsalam M, Rasker JJ. Antisynthetase syndrome complicating the course of established case with rheumatoid arthritis: a rare and under-recognized overlapping disease. Reumatol Clin. 2018; S1699258X(18)30116-5.

21. Giannini M, Notarnicola A, Dastmalchi M, et al. Heterogeneous clinical spectrum of interstitial lung disease in patients with anti-EJ anti-synthetase syndrome: a case series. Clin Rheumatol. 2016; 35: 2363-7.

22. Cavagna L, Castañeda S, Sciré C, GonzalezGay MA. Antisynthetase syndrome or what else? Different perspectives indicate the need for new classification criteria. Ann Rheum Dis. 2018; 77: 2018. 